\title{
Determinantes de las patentes y otras formas de propiedad intelectual de los estados mexicanos
}

\section{Determinants of patents and other forms of intellectual property of Mexican states}

\author{
Marco Antonio Almendarez Hernández*
}

\begin{abstract}
The objective of the paper is to econometrically evaluate the effects of scientific indicators and technological information on patents, utility models, and industrial designs. The theoretical and empirical knowledge production functions of Griliches (1979) and Hausman et al. (1984), respectively, were the methodology used as a reference. Panel count data at the level of the federative entity was the econometric treatment used to estimate the determinants of intellectual property. The findings revealed that Mexican states that devote more efforts to science and technological searches are more likely to produce intellectual property.
\end{abstract}

Keywords: patents, utility models, industrial designs, negative binomial model, panel data.

\section{Resumen}

El objetivo del trabajo fue evaluar econométricamente los efectos de indicadores científicos e información tecnológica sobre las patentes, los modelos de utilidad y los diseños industriales. Las funciones de producción de conocimiento teórica y empírica de Griliches (1979) y Hausman et al. (1984), respectivamente, fueron la metodología usada como referencia. Panel para datos de conteo a nivel de entidad federativa fue el tratamiento econométrico empleado para estimar los determinantes de la propiedad intelectual. Los hallazgos revelaron que los estados mexicanos que dedican más esfuerzos a la ciencia y las búsquedas tecnológicas son más propensos a producir propiedad intelectual.

Palabras Clave: patentes, modelos de utilidad, diseños industriales, modelo binomial negativo, datos de panel. 


\section{Introducción}

Recientemente, la propiedad intelectual ha intensificado el papel que juega en las economías para generar riqueza. Boldrin y Levine (2013) señalan que la formación de una relación más estrecha entre propiedad intelectual y prosperidad se debe a tres razones. En primer lugar, conforme se ha expandido la globalización, la creatividad y la innovación (propiedad intelectual como proxy de innovación) se han convertido en los pilares de la generación de valor agregado y, al mismo tiempo, han dotado de herramientas, de conocimientos y de habilidades al capital humano para elaborar productos o servicios en menor tiempo y con un mayor nivel de complejidad.

Otra explicación es que en el mandato presidencial de Reagan en los Estados Unidos, la protección de la propiedad intelectual cobró una gran relevancia y se fue propagando en el mundo con la fundación de la Organización Mundial de la Propiedad Intelectual (OMPI). La tercera razón es el surgimiento de un consenso entre las disciplinas de la economía y el derecho para argumentar la importancia de la existencia de un sistema de propiedad intelectual.

La OMPI (2015) señala que las principales formas de proteger toda creación de la mente humana son los derechos de autor, las patentes, los diseños industriales, los modelos de utilidad, las marcas y los secretos industriales. En especial, las patentes — como modalidades de propiedad intelectual - son indicadores de resultados que son considerados como proxy de la medición de la actividad innovadora o de innovación tecnológica (Pavitt, 1985; Griliches, 1990) y forman parte de una pequeña porción de este gran entramado; de la misma manera pertenecen a esta gama los modelos de utilidad (Kim et al., 2012) y los diseños industriales (Feeny y Rogers, 2003). Acorde con el Manual de Oslo (OCDE-Eurostat, 2006), existen cuatro formas de innovación que pueden surgir en las organizaciones: 1) Innovación en producto, 2) Innovación en proceso; 3 ) Innovación en mercadotecnia, 4) Innovación en organización.

La literatura moderna de crecimiento económico señala que la innovación es uno de los principales determinantes del crecimiento económico y provoca que las economías sean más prósperas (Romer, 1990; Grossman y Helpman, 1991; Aghion y Howitt, 1992). A nivel regional, tomando como unidad de análisis los estados o las municipalidades, existen trabajos que muestran que las regiones con mayor capacidad de innovación propagan externalidades tecnológicas, provocando que en los territorios beneficiados su crecimiento económico sea más rápido (Valdivia, 2007; Torres-Preciado et al., 2014). 
La propensión a proteger una innovación es de suma importancia porque, mediante este mecanismo legal, el poseedor de los derechos crea un ambiente de generación de incentivos para atraer inversiones privadas o públicas que fomenten las diferentes etapas de investigación y desarrollo, además, la participación en los derechos de propiedad intelectual mejora hasta lograr la meta del escalamiento comercial.

Usualmente la propiedad intelectual es considerada como uno de los resultados de investigación, desarrollo e innovación lograda en colaboración con la academia y el sector productivo; productos o procesos que se han protegido mediante algunas figuras de propiedad intelectual constituyen un punto de partida trascendental en uno de los eslabones del proceso de transferencia de tecnología, lo que facilita el curso del mismo (Rogers et al., 2000; Kurman, 2011; González-Pernía et al., 2013).

En un modelo lineal de producción de conocimiento y transformación de la innovación, las etapas iniciales de su desarrollo son consideradas básicas, con apoyo económico proveniente del sector público. En las fases intermedias, y con mayor nivel de desarrollo, los agentes económicos involucrados negocian la titularidad de la patente, la concesión de los derechos de explotación comercial y el financiamiento es realizado por capital de riesgo o capital ángel (Fundación Cotec, 2003).

Este funcionamiento es típico de los países desarrollados, ya que la mayor parte del gasto en investigación y desarrollo procede del sector privado, en tanto que en los países menos avanzados emana de fondos públicos (Martínez-Piva, 2009; Mendonca de, 2012). Las mejores prácticas y las lecciones aprendidas en países en desarrollo, entre ellos México, sugieren que los gobiernos deben enfocarse en invertir en ciencia básica, porque el fomento a esta actividad actúa como "semillero" de las innovaciones, mientras que la investigación aplicada y la comercialización debe financiarla el sector privado para lograr la eficiencia y mayor nivel de crecimiento económico (Finston, 2007).

En el caso de México, una parte del gasto en ciencia básica se orienta a pagar estímulos al personal científico, es el caso del Sistema Nacional de Investigadores (SNI), para incentivarlos constantemente a participar en diversas convocatorias con la finalidad de obtener financiamiento mediante concurso para realizar actividades científicas. Entre ellas se encuentra el desarrollo de artículos científicos, libros, capítulos de libro, congresos, formación de recursos humanos, el impacto de la difusión de sus trabajos plasmado en la generación de citas.

En particular, la literatura a nivel internacional y nacional ha mostrado que los artículos científicos, las citas, el capital humano perteneciente al sector de investigación y tecnológico, así como el gasto en investigación y desarrollo impactan positivamente sobre las patentes (Furman et al., 2002; 
Carayol, 2007; Stephan et al., 2007; Hernándezy Díaz, 2007; Hu y Mathews, 2008; Cowan y Zinovyeva, 2013; Calderón-Martínez y GarcíaQuevedo, 2013; Malva y Carree, 2013; Calderón-Martínez, 2014; Cozza y Schettino, 2015).

Por otra parte, los científicos, en el momento que empiezan a formar sus ideas sobre qué áreas investigar, o a medida que avanzan hacia otras fases de desarrollo como la investigación aplicada y obtienen resultados, requieren el apoyo de ciertas herramientas como las búsquedas de información tecnológica para saber si son susceptibles de protección mediante algunas formas de propiedad intelectual, conocer las tendencias tecnológicas del sector y cuáles son los principales competidores, estimar el valor de las patentes, optimizar los esfuerzos de investigación y desarrollo, evitar o anticipar conflictos relacionados a los derechos de propiedad (OMPI, 2013).

En particular, los sistemas de patentes constituyen la fuente de información tecnológica más consultada y analizada porque son la figura de propiedad intelectual más solicitada, ya que es una de las formas de protección más antiguas de las ideas y en la que los inventores pueden maximizar los beneficios de sus invenciones (Díaz, 2008).

El sistema de patentes tiene como principal función poner a disposición del público los documentos de patentes a escala mundial para que potencialicen los beneficios de su consulta (OMPI, 2013). De acuerdo con la Organización Mundial de Propiedad Intelectual (WIPO, 1999: 6-7), "los sistemas de patentes contribuyen al crecimiento y al desarrollo económico, creando las condiciones para el mercadeo y comercialización de las invenciones de distintas formas: a) Da un incentivo a la creación de una nueva tecnología, resultando en nuevos productos, oportunidades e invenciones; b) Contribuye a la creación de un entorno que facilita el éxito de la aplicación industrial de las invenciones y las nuevas tecnologías, y el marco jurídico, que fomenta la inversión, incluidos los de países extranjeros; $c$ ) Al publicar la información o nuevos inventos, actúa como un catalizador para la comercialización de invenciones y su transferencia al uso productivo; d) Es un instrumento de planeación estratégica comercial e industrial”.

En México, además de las patentes, existen otras formas de protección de propiedad intelectual, es el caso de los modelos de utilidad y los diseńos industriales. El IMPI (2017), para los tres tipos de protección, ofrece el servicio de búsquedas de información tecnológica y consiste en lo siguiente: 1) bibliográfica nacional, 2) bibliográfica internacional, 3) técnicas nacionales, 4) técnicas internacionales, 5) estados de la técnica, 6) información selectiva, 7) alerta tecnológica y 8) vigilancia tecnológica.

La importancia del vínculo entre las búsquedas de información tecnológica, el gasto en investigación y desarrollo y los productos científicos 
con las figuras de propiedad intelectual como las patentes, modelos de utilidad y los diseños industriales, así como la escasez de análisis empíricos en su conjunto en México - ya que solamente las publicaciones han girado alrededor de las patentes - han motivado la realización de este trabajo. Otro punto que también ha suscitado la elaboración del estudio es que en los manuscritos no se ha explorado la influencia de las búsquedas tecnológicas.

Las patentes, los modelos de utilidad y los diseños industriales se han elegido porque están estrechamente relacionadas con el know how o saber hacer de un proceso, formando parte sustantiva de una innovación, mientras que otras figuras como son las marcas y los derechos de autor tienen una relación distinta que no implica innovación en el proceso, producto o servicio. La hipótesis que se ha formulado en el trabajo es que las entidades federativas que ejecutan un mayor volumen de gasto en investigación y desarrollo, aquéllas que generan una mayor cantidad de productos científicos y registran más búsquedas tecnológicas, son más propensas a producir propiedad intelectual.

Esta investigación está dividida en cinco apartados; después de la introducción, la primera sección realiza una revisión de la literatura a nivel internacional y nacional sobre los factores que se relacionan con la propiedad intelectual, clasificando los estudios por país, estructura de datos y la técnica econométrica. La segunda sección describe y analiza la situación de la producción de las figuras de propiedad intelectual y cómo se relaciona su generación con los investigadores y las universidades. La tercera sección expone el planteamiento del modelo teórico y empírico usado para cumplir con el objetivo. En la cuarta sección se muestran y se discuten los resultados. Finalmente, en la última sección, se describen las conclusiones del trabajo.

\section{Revisión de la literatura}

A principios del siglo XXI el patentamiento experimentó un auge en economías avanzadas como Estados Unidos, Japón y algunos países de Europa, apoyado en formas de organización por medio de redes de conocimiento y mercado. Durante este periodo, las empresas requirieron $-\mathrm{y}$ actualmente siguen demandando- más patentes para establecer más lazos de cooperación, ser entes más competitivos, lo que se traduce en disminución de los costos de producción, generación de productos de más calidad, exigencias que cada vez son mayores en los mercados globalizados (OCDE, 2004).

Este tipo de protección es aplicado generalmente en economías que desarrollan innovaciones "de gran avance" (breakthrough), como en la rama de la biotecnología y la farmacéutica. En naciones donde predominan las 
innovaciones incrementales como la industria del transporte, la óptica, la electrónica básica y artículos domésticos se sugiere protegerlos por la figura de modelo de utilidad, el cual es más característico de países menos desarrollados y constituye una alternativa para promover la innovación y el crecimiento económico.

Solamente algunos países, incluyendo México, reconocen esta modalidad. Algunas de las ventajas de los modelos de utilidad respecto de las patentes son: $a$ ) Los costos son más bajos; $b$ ) Pocos requisitos para mostrar la novedad; $c$ ) Pocos requisitos para mostrar el carácter de inventiva; d) Procedimiento de registro es menos complicado. Las desventajas son las siguientes: $a$ ) No es posible proteger métodos o procesos; $b$ ) La vigencia de la protección es de 10 años; c) Es más débil que una patente (Brack, 2009; Suthersanen, 2006).

Los diseños industriales son usados cuando se crea un diseño para un producto existente, como los dibujos y modelos industriales, los cuales para otorgar su protección deben cumplir con los requisitos de novedad y aplicación industrial (IMPI, 2018). Una patente es más valorada porque su utilidad es el componente más complicado de crear.

Estas son algunas razones por las cuales la literatura se ha centrado en analizar los factores que han influenciado las patentes, es pionero el estudio de Hausman et al. (1984), en el cual relacionó esta variable dependiente con el gasto en investigación y desarrollo, estimando esta función con el tratamiento de un modelo econométrico en el contexto de panel de efectos fijos con datos de conteo.

En el cuadro 1 se muestra la evidencia internacional que se ha realizado sobre los efectos de la propiedad intelectual. Es importante recalcar que no es la pretensión realizar una búsqueda exhaustiva, sino que se exponen algunos de los trabajos más recientes. El tipo de datos van desde sección cruzada hasta datos de panel, con técnicas econométricas desde las más elementales como mínimos cuadrados ordinarios (OLS por sus siglas en inglés) hasta las más sofisticadas como datos de conteo (las cuales son de las más usadas), variables instrumentales (IV por sus siglas en inglés), mínimos cuadrados en dos etapas (2SLS por sus siglas en inglés), efectos fijos, efectos aleatorios, método generalizado de los momentos (GMM por sus siglas en inglés), modelo de selección de muestras Heckman, Regresión de Umbral Uniforme de Panel.

Las variables independientes consideradas son macroeconómicas, institucionales, microeconómicas, indicadores de ciencia y tecnología, demográficos, salud, transporte, geográficos, gobernanza, efectos spillovers, índices de densidad clique, índices de proximidad social interno y externos, índices de proximidad, índices de densidad de inventor. La elaboración de las investigaciones nacionales son actuales y la mayor parte se 


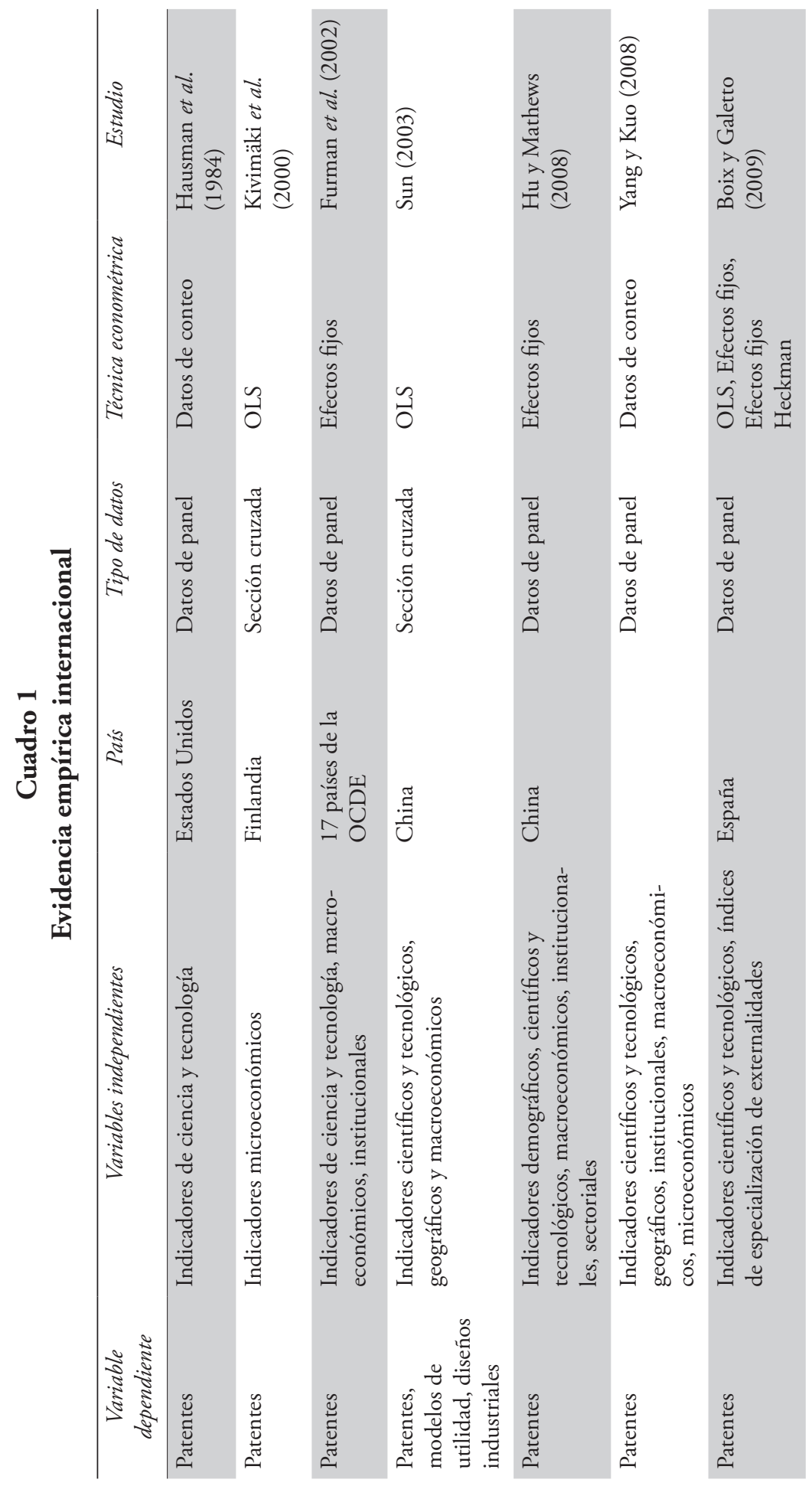




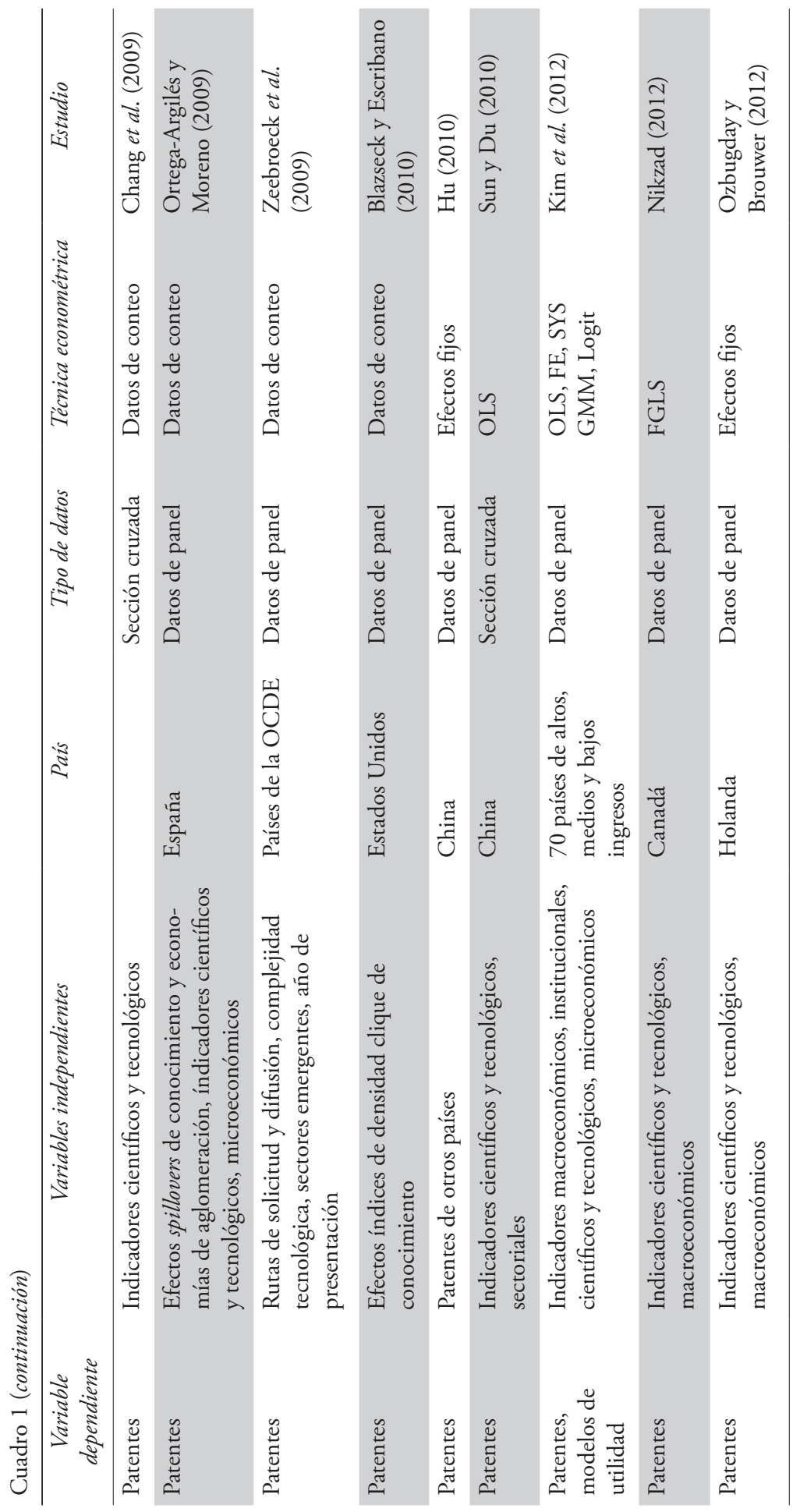




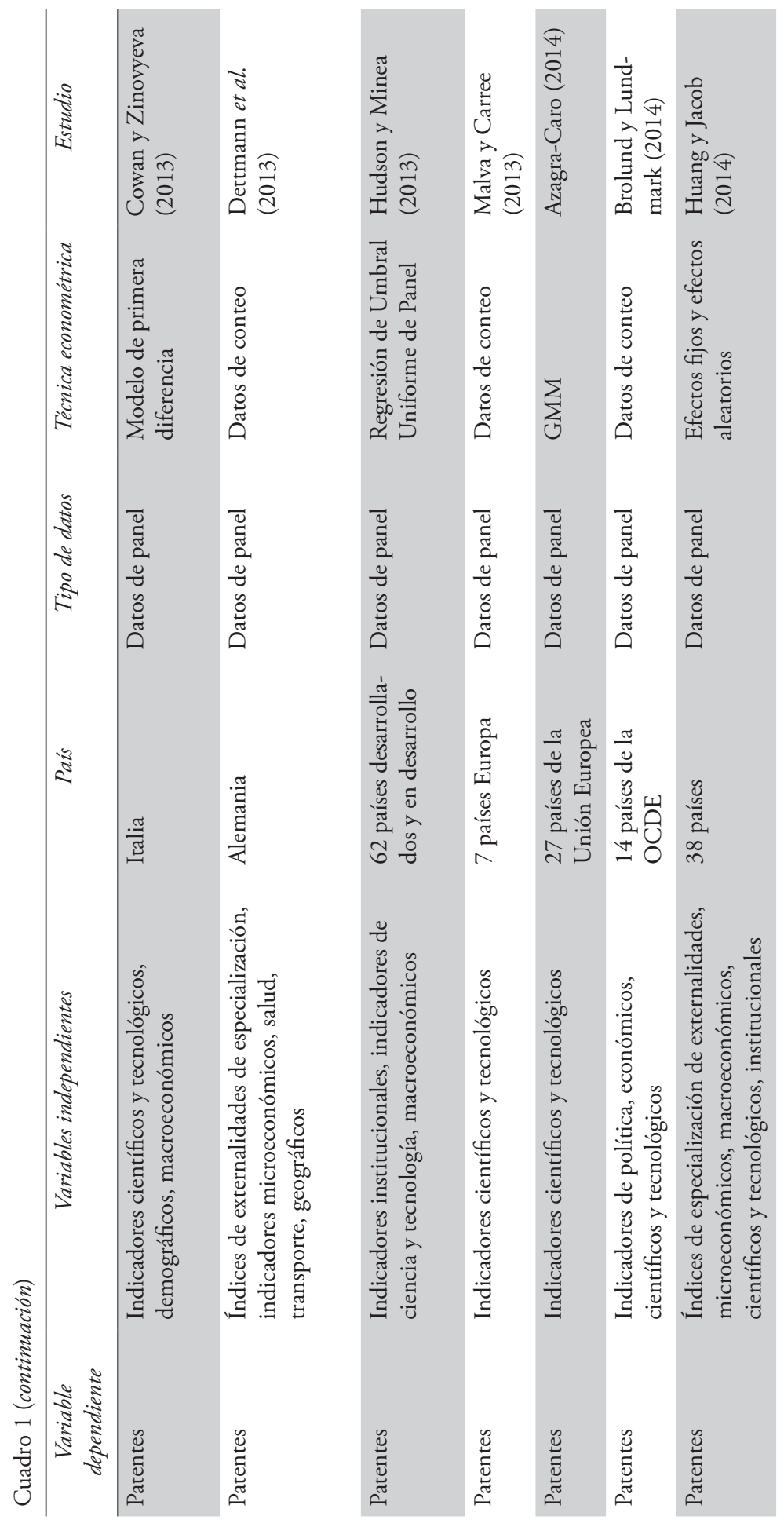




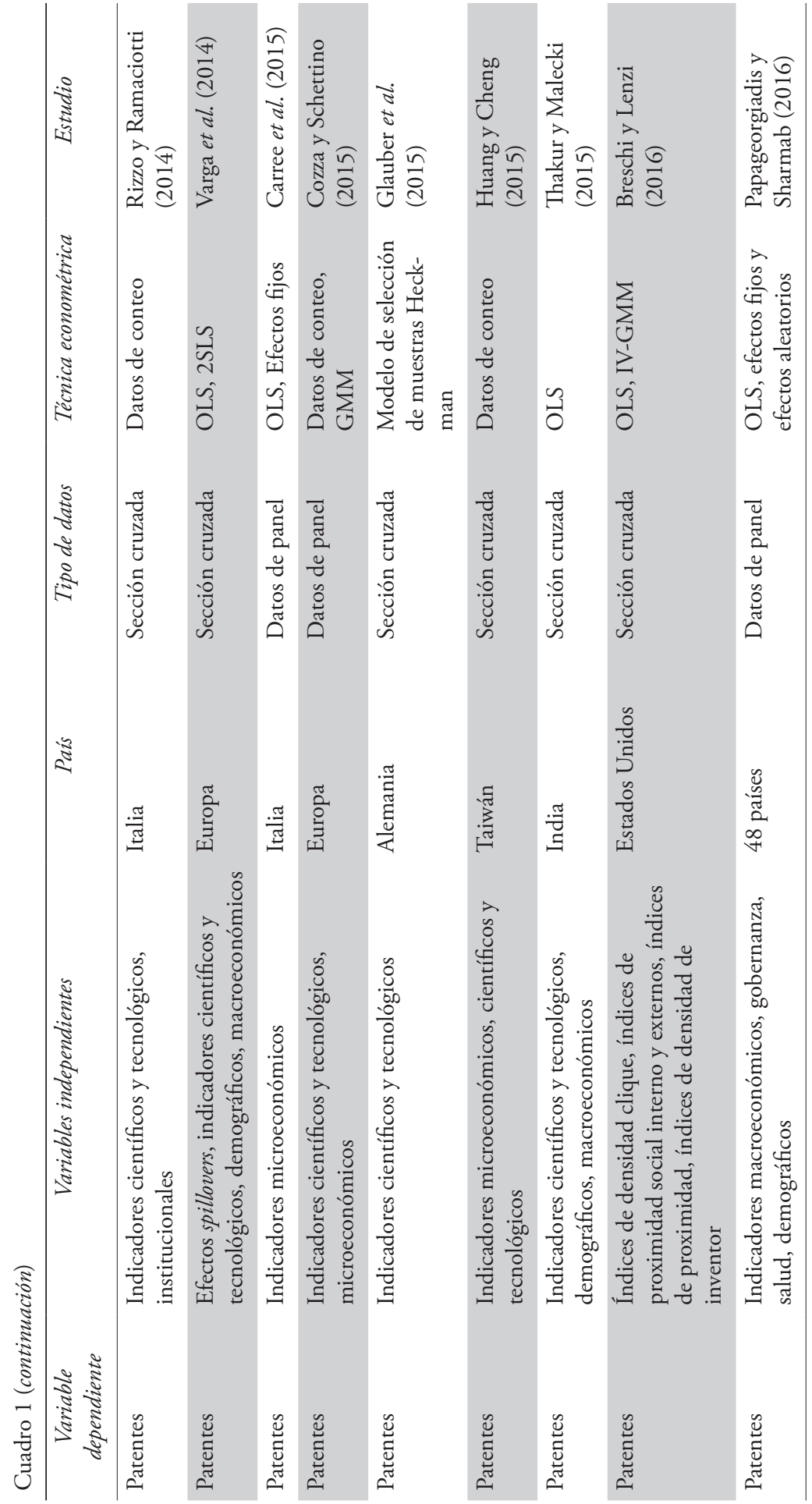




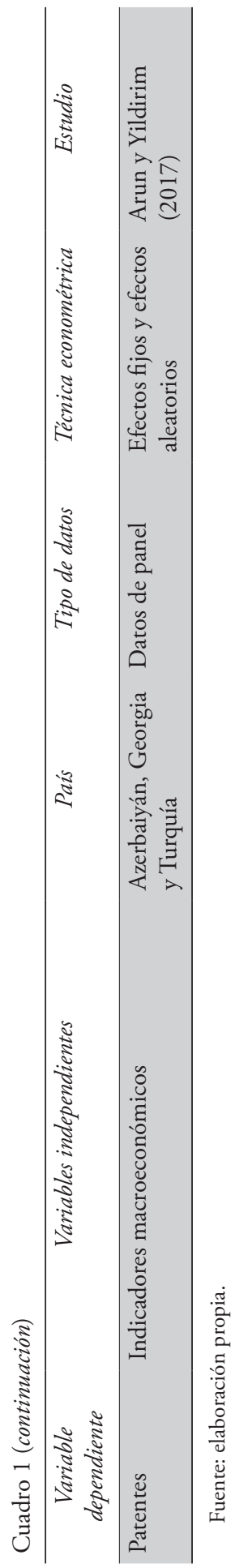


concentra en las patentes con datos de panel y con modelos econométricos de datos de conteo (cuadro 2).

\section{Cuadro 2}

\section{Estudios nacionales}

\begin{tabular}{|c|c|c|c|c|}
\hline $\begin{array}{c}\text { Variable } \\
\text { dependiente }\end{array}$ & $\begin{array}{c}\text { Variables } \\
\text { independientes }\end{array}$ & $\begin{array}{l}\text { Tipo } \\
\text { de datos }\end{array}$ & $\begin{array}{c}\text { Técnica } \\
\text { econométrica }\end{array}$ & Estudio \\
\hline Patentes & $\begin{array}{l}\text { Indicadores macroeco- } \\
\text { nómicos, científicos y } \\
\text { tecnológicos }\end{array}$ & $\begin{array}{l}\text { Datos de } \\
\text { panel }\end{array}$ & GLS & $\begin{array}{l}\text { Hernández y } \\
\text { Díaz (2007) }\end{array}$ \\
\hline Patentes & $\begin{array}{l}\text { Indicadores macroeco- } \\
\text { nómicos, demográfi- } \\
\text { cos }\end{array}$ & $\begin{array}{l}\text { Datos de } \\
\text { panel }\end{array}$ & $\begin{array}{l}\text { Datos de } \\
\text { conteo }\end{array}$ & $\begin{array}{l}\text { Germán-Soto et } \\
\text { al. (2009) }\end{array}$ \\
\hline Patentes & $\begin{array}{l}\text { Indicadores científicos } \\
\text { y tecnológicos }\end{array}$ & $\begin{array}{l}\text { Datos de } \\
\text { panel }\end{array}$ & $\begin{array}{l}\text { Datos de } \\
\text { conteo }\end{array}$ & $\begin{array}{l}\text { Calderón-Martí- } \\
\text { nez y García- } \\
\text { Quevedo (2013) }\end{array}$ \\
\hline Patentes & $\begin{array}{l}\text { Indicadores científicos } \\
\text { y tecnológicos }\end{array}$ & $\begin{array}{l}\text { Datos de } \\
\text { panel }\end{array}$ & $\begin{array}{l}\text { Datos de } \\
\text { conteo }\end{array}$ & $\begin{array}{l}\text { Calderón-Martí- } \\
\text { nez (2014) }\end{array}$ \\
\hline Patentes & $\begin{array}{l}\text { Indicadores de } \\
\text { educación, científicos } \\
\text { y tecnológicos, } \\
\text { macroeconómicos, } \\
\text { coeficientes de } \\
\text { localización, efectos } \\
\text { espaciales }\end{array}$ & $\begin{array}{l}\text { Datos de } \\
\text { panel }\end{array}$ & Efectos fijos & $\begin{array}{l}\text { Germán-Soto y } \\
\text { Gutiérrez (2015) }\end{array}$ \\
\hline Patentes & $\begin{array}{l}\text { Stock de conocimien- } \\
\text { tos }\end{array}$ & $\begin{array}{l}\text { Serie de } \\
\text { tiempo }\end{array}$ & OLS & $\begin{array}{l}\text { Gracia y } \\
\text { Moctezuma } \\
(2016)\end{array}$ \\
\hline Patentes & $\begin{array}{l}\text { Indicadores científicos } \\
\text { y tecnológicos }\end{array}$ & $\begin{array}{l}\text { Sección } \\
\text { cruzada }\end{array}$ & $\begin{array}{l}\text { Datos de } \\
\text { conteo }\end{array}$ & $\begin{array}{l}\text { Guzman et al. } \\
\text { (2016) }\end{array}$ \\
\hline
\end{tabular}

Fuente: elaboración propia.

Un importante punto a mencionar es que la asociación entre las tres formas de propiedad intelectual y las búsquedas de información tecnológica ha sido poco explorada a nivel internacional, en México, particularmente, no se ha realizado.

En específico, los sistemas de patentes tienen como función primordial indagar en la información tecnológica y realizar su correspondiente análisis, así como brindar dichos análisis al público; los sistemas de patentes son en su naturaleza de componentes sustanciales de las fases de la planeación estratégica para la creación de nuevos productos y procesos tecnológicos (Lee et al., 2008; Carvalho et al., 2013), con el desarrollo de los sistemas algunas formas de innovación pueden manifestarse en las 
organizaciones y es posible detectar aquellos rasgos con potencial, de posicionamiento en el mercado y conseguir ganancias más altas (Livotov, 2015; Jeong y Yoon, 2015).

Sin embargo, Baglieri y Cesaroni (2013) consideran que los sistemas de patentes deben orientarse hacia una visión más integral, para que a los actores del mercado de capital y del sector público les proporcionen elementos ligados a la disminución del riesgo de la inversión hecha en el desarrollo futuro de las patentes.

La conformación y la gestión de los sistemas de patentes, así como otra estructura de sistema de información tecnológica, han contribuido históricamente como potencial referente en la investigación del desarrollo de nuevos productos (Nambisan, 2003). Los negocios son favorecidos con el uso de la información tecnológica en los eslabones de los procesos de producción, comercialización, distribución y planeación.

Un estudio elaborado en Irán (Foukolaei et al., 2018) sobre el nexo entre la capacidades de información tecnológica y el desempeño en la industria farmacéutica a nivel organizacional, basado en una encuesta realizada a los departamentos de gerencia de ventas, producción, mercadotecnia y recursos humanos, con una muestra de 170 empresas, encontró que mejora la eficiencia de la cadena de suministro respecto a pureza, agilidad y resiliencia, y estos mismos atributos, acrecientan el desempeño.

Otra investigación efectuada en Estados Unidos (DeGroote y Marx, 2013), sobre el vínculo entre la información tecnológica y la cadena de suministro con datos de 193 compañías del sector manufacturero, exhibió que esta última mejora su capacidad en la lectura de los cambios ocurridos en el mercado, beneficiando las ventas, la satisfacción al cliente, la velocidad de comercialización, la rentabilidad y la participación en el mercado, la reducción de costos y, en la sección de planeación, hay una mayor conjunción en la construcción y puesta en marcha de proyectos.

En este sentido, los sistemas de patentes, como cualquier otro tipo de información tecnológica, son necesarios para producir propiedad intelectual, porque son concebidos como un conjunto de herramientas esenciales que sirven de guía sobre la forma de decidir qué productos, métodos o procesos tienen potencialidad y en qué momento deben lanzarse al mercado, previniendo el ahorro de costos y tiempo; asimismo, monitorean la capacidad de inventiva para obtener un dictamen con mayor certeza sobre qué procesos, métodos o productos pueden protegerse con derechos de propiedad intelectual o representan una referencia útil para que quienes toman las decisiones tengan elementos suficientes para elegir invenciones de tipo incremental, radical y disruptiva (WIPO, 1999; Díaz, 2008; OMPI, 2013). 


\section{Relación entre patentes, otras formas de propiedad intelectual e impacto científico}

La escasa inversión en ciencia y tecnología, el bajo nivel de protección en propiedad intelectual, así como diversos problemas estructurales, sociales y culturales son características heterogéneas de los países latinoamericanos y menos avanzados, y son algunos de los factores por los cuales no son economías basadas en conocimiento. En México —aunque en los últimos años se ha promulgado en 2002 y se ha modificado la ley de ciencia y tecnología en 2009 y se han promovido políticas públicas para generar una mayor participación y vinculación entre los sectores productivo, social público y académico - los resultados en materia de solicitudes de las diferentes modalidades de propiedad intelectual ante el Instituto Mexicano de la Propiedad Industrial (IMPI) son bajos en comparación a países más avanzados (IMPI, 2017).

Es importante resaltar que en México los diseños industriales concentran el mayor volumen de solicitudes de propiedad intelectual respecto al carácter inventivo, representaron entre $65 \%$ y $75 \%$ en el periodo 2003 2012. Las solicitudes de patentes ocupan el segundo lugar, registran una oscilación entre $14.5 \%$ y $22 \%$. (IMPI, 2017). Las solicitudes de modelos de utilidad se ubican en la última posición, con una fluctuación entre $7.5 \%$ y $15 \%$; comparado con países más avanzados, los registros en propiedad intelectual de las universidades nacionales ante el IMPI son bajos, un ejemplo de ello es constatado por los registros de patentes de las empresas transnacionales en el sector biofarmacéutico, las cuales equivalen al 98\% (García, 2017).

De acuerdo con Calderón-Martínez (2014), las universidades desempeñan una función sustancial en los cimientos de la investigación y conocimientos especializados; aquéllas que han tenido la capacidad para atender las necesidades del sector productivo han sido exitosas en la transferencia del conocimiento, principalmente este patrón de comportamiento se ha manifestado en las instituciones públicas, a tal grado que concentran $95 \%$ de las patentes concedidas.

Sin embargo, el volumen de solicitudes de propiedad intelectual es incipiente por las disparidades entre demandas de las distintas ramas de la economía y las investigaciones, la incertidumbre de los resultados del escalamiento comercial y la orientación de las universidades de desarrollar ciencia enfocada al bienestar social y cultural, es decir, una visión y misión integral, no como universidades emprendedoras o encaminadas al mercado. La mayor propensión a patentar en universidades públicas es un reflejo de que el gasto en $\mathrm{I}+\mathrm{D}$ nacional proviene de origen público, siendo mayor a 50\% (OCDE, 2015). 
Otra explicación de la baja proporción en que se protege la propiedad intelectual en las universidades también es corroborada a través de los afiliados al SNI. Para mantener su membresía dentro del sistema requieren competir intensamente por proyectos en distintas convocatorias con la intención de obtener financiamiento de origen principalmente público, debido a que continuamente están sujetos a evaluación para ingresar, permanecer o promocionarse. Los recursos les permiten desarrollar una serie de productos como artículos científicos, libros, capítulos de libro, citas a los mismos o algún otro producto que surja de algunos resultados de sus investigaciones con el propósito de acumularlos en su periodo de membresía y reportarlos ante la comisión dictaminadora, que se encargará de emitir la evaluación.

Amigo (2009) señala que el incremento de la productividad de los investigadores apegados a este lineamiento es el factor clave para que ingresen, permanezcan o se promuevan a rangos más altos del SNI y no obedece a la solicitud de patentes; además, el tiempo que tardan los árbitros en dictaminar un artículo y la editorial en publicarlo es menor al de la concesión de las patentes que es alrededor de cuatro años, ya que demanda un examen riguroso por parte del Instituto Mexicano de la Propiedad Industrial (IMPI).

Los modelos de utilidad son concedidos aproximadamente en un intervalo de año y medio y cuatro años, y los diseños industriales en un lapso de seis meses a cuatro ańos. Por estas razones, Ulku (2004) recomienda utilizar las patentes solicitadas cuando es grande la longitud de la brecha en que tarda en concederse una patente. En este mismo sentido, también se sugiere usar los modelos de utilidad y los diseńos industriales solicitados.

Adicionalmente, García (2017) sugiere que las universidades no deben preocuparse por poner especial atención en obtener indicadores de rentabilidad altos como el valor presente neto, la tasa interna de retorno, el periodo de recuperación de la inversión y el rendimiento sobre la inversión para patentar vía el licenciamiento, sino que requieren realizarlo para mostrar el respaldo en capital humano e investigación y desarrollo con el que cuentan las instituciones, el cual bajo estas condiciones genera un ambiente de prestigio y confianza para que el sector privado se incentive en invertir en las innovaciones. Además, las patentes no son la única forma de transferir el conocimiento sino que se puede realizar también con los otros tipos de propiedad intelectual que se han mencionado. 


\section{Modelo teórico y especificación econométrica de la función de producción de propiedad intelectual}

El estudio se sustenta en la formulación teórica de Griliches (1979) en la que establece una función producción de conocimiento o de ideas que, desde la perspectiva empírica (Hausman et al., 1984), usa como indicador las patentes; como modalidad de propiedad intelectual tiene como objeto proteger legalmente y valorizar económicamente la actividad innovadora que se genera en los diversos sectores productivos. La ecuación se deriva de una función producción que depende de una serie de insumos denotada de la forma siguiente:

$$
Y=F(X, K, u)
$$

Donde $X$ es un índice de insumos convencionales como Trabajo y Capital, $K$ es una medida del Estado actual del conocimiento técnico, $u$ representa todos los demás determinantes no medidos de producción y productividad. El modelo original se fundamenta en una relación entre el nivel de conocimiento tecnológico y el gasto en investigación y desarrollo actuales y sus valores pasados. Se apoya en los supuestos siguientes: 1) Existe separabilidad de los insumos convencionales de los valores actuales y rezagados del gasto en investigación y desarrollo. 2) Si se establece una función lineal de los retardos de la serie de gasto en investigación y desarrollo, conlleva a que no se generen rendimientos decrecientes a escala. Por lo que el modelo se representa de la forma siguiente,

$$
K=G[W(B) R, v]
$$

Donde $X$ son influencias no medidas del capital acumulado de conocimiento $y$

$$
W(B) R=\left(w_{0}+w_{1} B+w_{2} B_{2}+\ldots\right) R_{t}=w_{0} R_{t}+w_{1} R_{t-1}+w_{2} R_{t-2}+\ldots
$$

Donde $R$ es el gasto en investigación y desarrollo.

La función producción de conocimiento es modificada y adaptada para tomar en cuenta otras variables que afectan la actividad innovadora. Esta propuesta se basa en los trabajos empíricos nacionales e internacionales como los de Furman et al. (2002), Carayol (2007), Stephan et al. (2007), Hernández y Díaz (2007) Hu y Mathews (2008), Cowan y Zinovyeva (2013), Calderón-Martínez y García-Quevedo (2013) Malva y Carree (2013), Calderón-Martínez (2014), Cozza y Schettino (2015), 
que han incorporado como factores que influyen en la innovación (patentes), además del gasto en investigación y desarrollo como serie actual, el personal involucrado en el sector científico y tecnológico, las publicaciones científicas y las citas a las mismas.

Como aportación al análisis son introducidas como factor las búsquedas de información tecnológica, las cuales se han abordado poco a nivel internacional; además en México no se han realizado estudios sobre los determinantes de la propiedad intelectual como los modelos de utilidad y los diseños industriales y mucho menos se ha estudiado el efecto de la información tecnológica. Las funciones producción de ideas personificadas por los tres tipos de propiedad intelectual se definen por:

$$
\begin{aligned}
& P=F(I T, G D, S N I, A R T, C I T) \\
& M=F(I T, G D, S N I, A R T, C I T) \\
& D=F(I T, G D, S N I, A R T, C I T)
\end{aligned}
$$

La descripción de las variables se presenta en el cuadro 3. La información está estructurada en datos de panel a nivel de entidad federativa, abarcando un periodo de 2000 a 2012.

Dada la naturaleza expresada como datos de recuento de las tres figuras de propiedad intelectual, los modelos de regresión para datos de conteo son la elección econométrica adecuada para estimar las funciones, el modelo Poisson es usado cuando la variable dependiente está denominada en valores enteros no negativos. La variable propiedad intelectual puede tomar valores de 0 a $\infty$. La función de densidad de probabilidad Poisson se escribe como:

$$
\begin{gathered}
\operatorname{Pr}\left(P_{i t}=p_{i t} \mid X_{i t}\right)=\frac{\varepsilon-\lambda_{i t} \lambda_{i t}{ }_{i t}}{p_{i t}}, n=0,1,2 \ldots \\
\operatorname{Pr}\left(M_{i t}=m_{i t} \mid X_{i t}\right)=\frac{\varepsilon-\lambda_{i t \lambda_{i t}{ }^{i t}}}{m_{i t}}, n=0,1,2 \ldots \\
\operatorname{Pr}\left(D_{i t}=d_{i t} \mid X_{i t}\right)=\frac{\varepsilon-\lambda_{i t \lambda_{i t}{ }_{i t}}}{d_{i t}}, n=0,1,2 \ldots
\end{gathered}
$$


donde

$\lambda_{i t}=\exp \left(x_{i t} \beta+\varepsilon_{i}\right)=\exp \left(\beta_{0}+\beta_{1} I T_{i t}+\beta_{2} \ln G D_{i t}+\beta_{3} \ln S N I_{i t}+\beta_{4} \ln A R T_{i t}+\right.$ $\left.\beta_{5} \ln C I T_{i t}+\varepsilon_{i}\right)$

Por tratarse de datos de panel, una de las ventajas de su uso es que puede capturar la heterogeneidad no observada, conformada por la representación de características que diferencian a los entes solicitantes que acuden a las diferentes formas de propiedad intelectual para proteger sus invenciones de cada entidad federativa, las cuales son desconocidas por el investigador; esta misma puede ser introducida como un efecto específico no observado dentro de $\lambda_{i t}$ y puede ser tratado como fijo o aleatorio dependiendo del tratamiento econométrico. La ventaja de efectos aleatorios en relación a efectos fijos es que si está correctamente especificado es más eficiente. Por otra parte, la ventaja de efectos fijos con respecto

\section{Cuadro 3}

\section{Descripción de las variables}

\begin{tabular}{|c|c|c|}
\hline Variable & Descripción & Fuente \\
\hline $\mathrm{P}$ & $\begin{array}{l}\text { Número de solicitudes de patentes de la } \\
\text { entidad federativa } i \text { en el tiempo } t\end{array}$ & $\begin{array}{l}\text { Instituto Mexicano de la Pro- } \\
\text { piedad Industrial }\end{array}$ \\
\hline M & $\begin{array}{l}\text { Número de solicitudes de modelos de utili- } \\
\text { dad de la entidad federativa } i \text { en el tiempo } t\end{array}$ & $\begin{array}{l}\text { Instituto Mexicano de la Pro- } \\
\text { piedad Industrial }\end{array}$ \\
\hline $\mathrm{D}$ & $\begin{array}{l}\text { Número de solicitudes de diseños industria- } \\
\text { les de la entidad federativa } i \text { en el tiempo } t\end{array}$ & $\begin{array}{l}\text { Instituto Mexicano de la Pro- } \\
\text { piedad Industrial }\end{array}$ \\
\hline IT & $\begin{array}{l}\text { Número de búsquedas de información } \\
\text { tecnológica de la entidad federativa } i \text { en el } \\
\text { tiempo } t\end{array}$ & $\begin{array}{l}\text { Instituto Mexicano de la Pro- } \\
\text { piedad Industrial }\end{array}$ \\
\hline $\ln G D$ & $\begin{array}{l}\text { Logaritmo natural del gasto en investigación } \\
\text { y desarrollo de la entidad federativa } i \text { en el } \\
\text { tiempo } t\end{array}$ & $\begin{array}{l}\text { Consejo Nacional de Ciencia } \\
\text { y Tecnología }\end{array}$ \\
\hline $\operatorname{lnSNI}$ & $\begin{array}{l}\text { Logaritmo natural del número de miembros } \\
\text { en el Sistema Nacional de Investigadores de } \\
\text { la entidad federativa } i \text { en el tiempo } t\end{array}$ & $\begin{array}{l}\text { Consejo Nacional de Ciencia } \\
\text { y Tecnología }\end{array}$ \\
\hline ART & $\begin{array}{l}\text { Número de artículos científicos publicados } \\
\text { de la entidad federativa } i \text { en el tiempo } t\end{array}$ & Atlas de la Ciencia Mexicana \\
\hline CIT & $\begin{array}{l}\text { Número de citas a los artículos científicos } \\
\text { publicados de la entidad federativa } i \text { en el } \\
\text { tiempo } t\end{array}$ & tlas de la Ciencia Mexicana \\
\hline
\end{tabular}

Fuente: elaboración propia. 
a efectos aleatorios es que no requiere que se establezca suposición sobre la distribución poblacional de $\varepsilon_{i}$.

La forma que asuma la distribución del modelo Poisson va a depender de la opción que se escoja de la distribución de probabilidad del componente estocástico. Por los supuestos en los que se fundamenta, lo convierten en un modelo muy restrictivo: 1) Para realizar inferencias válidas sobre los parámetros es necesario verificar la propiedad consistente en la igualdad entre la media y la varianza de $\lambda_{i t}$, conocida como equidispersión e implica la dificultad para obtener un buen ajuste. 2) La base de datos usada puede contener en exceso valores de cero el cual no es consistente con el modelo. 3) Cuando la información estadística está construida en ambiente de datos de panel, si no se capta la heterogeneidad no observada mediante la incorporación de efectos específicos individuales, puede ocurrir sobredispersión. Cameron y Trivedi (2005) señalan que el incumplimiento de estas propiedades conlleva a la sobredispersión (la varianza es mayor que la media) o infradispersión (la varianza es menor que la media).

La sobredispersión es común en la base empírica y de acuerdo con Winkelmann y Zimmermann (1991), una forma de considerar su presencia es modelando la varianza en función de la media de la siguiente manera:

$$
\operatorname{Var}\left(i p_{i t} \mid x_{i t}\right)=\left(\sigma^{2}-1\right)\left[E\left(i p_{i t} \mid x_{i t}\right)\right]^{k+1}+E\left(i p_{i t} \mid x_{i t}\right)
$$

Donde ip representa las modalidades de propiedad intelectual, $\sigma^{2}$ es el parámetro de dispersión y $k$ es un parámetro de no linealidad y son independientes de $\beta$. Si $\sigma^{2}=1$, existe equidispersión y la especificación apropiada es el modelo Poisson; si $\sigma^{2}>1$, indica sobredispersión, es conveniente seleccionar el modelo binomial negativo el cual generalmente es el más preferido. Cuando los coeficientes asociados a las variables independientes se estiman con efectos aleatorios, $\varepsilon_{i}$ sigue una distribución gamma y este componente capta errores de especificación como variables no observadas y mediciones de la base de datos; si $0<\sigma^{2}<1$, advierte infradispersión y es apto el modelo de binomial de parámetro continuo, el cual no es muy frecuente.

\section{Análisis de los resultados}

Para establecer una comparación entre el modelo Poisson y el modelo binomial negativo, y elegir el apropiado, se tomaron en cuenta las distribuciones de frecuencias de cada forma de propiedad intelectual, la estadística descriptiva y medidas de ajuste como el Criterio de Información de Akaike 
(AIC por sus siglas en inglés) y el Criterio de Información Bayesiano (BIC por sus siglas en inglés). El número total de ceros de patentes fue de 34 y representa $8.17 \%$; los modelos de utilidad registraron 53 ceros y constituyen $12.74 \%$; los diseños industriales contaron con 80 ceros y equivale al $19.23 \%$ (cuadro 4). Se puede apreciar que en las tres modalidades la varianza es mayor que la media (cuadro 5), manifestando la existencia de sobredispersión. Adicionalmente, se calcularon los estadísticos AIC y el BIC, revelando valores más bajos en el modelo binomial negativo que en el Poisson, sugiriendo que la especificación correcta es el primero.

Se efectúo una prueba de razón de verosimilitud que contrasta la estructura de datos de panel de efectos aleatorios con la conformación agrupada, la cual es parecida a la prueba del multiplicador de Lagrange (LM) de Breusch y Pagan y que es utilizada en datos de panel lineales. Para todas las regresiones hechas, favorece la conformación de un panel.

\section{Cuadro 4}

Distribución de frecuencias de la propiedad intelectual

\begin{tabular}{|c|c|c|c|}
\hline \multirow[t]{2}{*}{ Categoría } & \multicolumn{3}{|c|}{ Conteo } \\
\hline & Patentes & Modelos de utilidad & Diseños industriales \\
\hline 0 & 34 & 53 & 80 \\
\hline 1 & 43 & 44 & 50 \\
\hline 2 & 34 & 41 & 25 \\
\hline 3 & 33 & 41 & 27 \\
\hline 4 & 25 & 31 & 21 \\
\hline 5 & 23 & 30 & 17 \\
\hline 6 & 13 & 21 & 11 \\
\hline 7 & 22 & 17 & 16 \\
\hline 8 & 13 & 17 & 11 \\
\hline 9 & 7 & 10 & 6 \\
\hline 10 & 14 & 17 & 7 \\
\hline de 11 a 20 & 56 & 30 & 36 \\
\hline de 21 a 40 & 40 & 28 & 40 \\
\hline de 41 a 60 & 21 & 15 & 6 \\
\hline de 61 a 80 & 14 & 7 & 3 \\
\hline de 80 a 100 & 7 & 2 & 11 \\
\hline más de 100 & 17 & 12 & 49 \\
\hline Total & 416 & 416 & 416 \\
\hline
\end{tabular}

Fuente: elaboración propia con base en información proporcionada por el IMPI. 


\section{Cuadro 5}

Estadística descriptiva

\begin{tabular}{lrrrr}
\hline \multicolumn{1}{c}{ Variable } & \multicolumn{1}{c}{ Media } & Desviación estándar & Minimo & \multicolumn{1}{c}{ Máximo } \\
\hline $\mathrm{P}$ & 21.8414 & 46.1626 & 0 & 426 \\
$\mathrm{M}$ & 12.7933 & 24.3324 & 0 & 162 \\
$\mathrm{D}$ & 35.7524 & 80.1673 & 0 & 610 \\
$\mathrm{IT}$ & 54.1625 & 178.3174 & 0 & 1309 \\
$\mathrm{lnGD}$ & 17.9434 & 1.4535 & 13.4970 & 22.4285 \\
lnSNI & 5.2872 & 1.1283 & 2.6391 & 8.8324 \\
ART & 536.4281 & 1551.4750 & 1 & 10759 \\
$\mathrm{CIT}$ & 3665.4020 & 10401.3100 & 1 & 71184 \\
\hline
\end{tabular}

Fuente: elaboración propia.

La prueba de Hausman fue realizada para comparar entre efectos aleatorios y efectos fijos, indicando para todas las estimaciones que no se rechaza la hipótesis nula de que no existe correlación entre los efectos individuales y las variables explicativas, por lo que el primer método es consistente y eficiente (cuadros 6, 7 y 8 ).

En este sentido, el modelo de efectos aleatorios fue el tratamiento econométrico correcto y su estimación indica que se está tomando en cuenta el efecto específico individual, personificado en las entidades federativas.

Las entidades federativas en su conjunto tienen una media común para la intersección, y las diferencias entre cada una de ellas están expresadas en la variación de sus intersecciones, midiéndose por el término de error, pero los coeficientes de la regresión son constantes.

En el modelo de efectos fijos, las intersecciones de cada una de las entidades federativas varían, diferenciándolas entre ellas, y también los coeficientes de la regresión son constantes (Gujarati, 2004: 640-649). En México, de acuerdo a lo encontrado por Germán-Soto y Gutiérrez (2015), la variación de los coeficientes entre entidad federativa y en el tiempo no son adecuados.

La búsqueda de información tecnológica fue estimada por separado debido a que se encontraron problemas de multicolinealidad con el resto de las variables, ocasionando distorsiones en los estimadores. Los indicadores usados para detectarla son los sugeridos por la literatura como el factor de inflación de la varianza (FIV) y el número de condición. El primer criterio arrojó valores superiores a 10, los cuales no se consideran aceptables. El segundo índice reveló valores por arriba de 20, punto de referencia que no debe excederse para ser admisible (Greene, 2003). El gasto en investigación y desarrollo y el SNI fueron disgregados de los demás por 


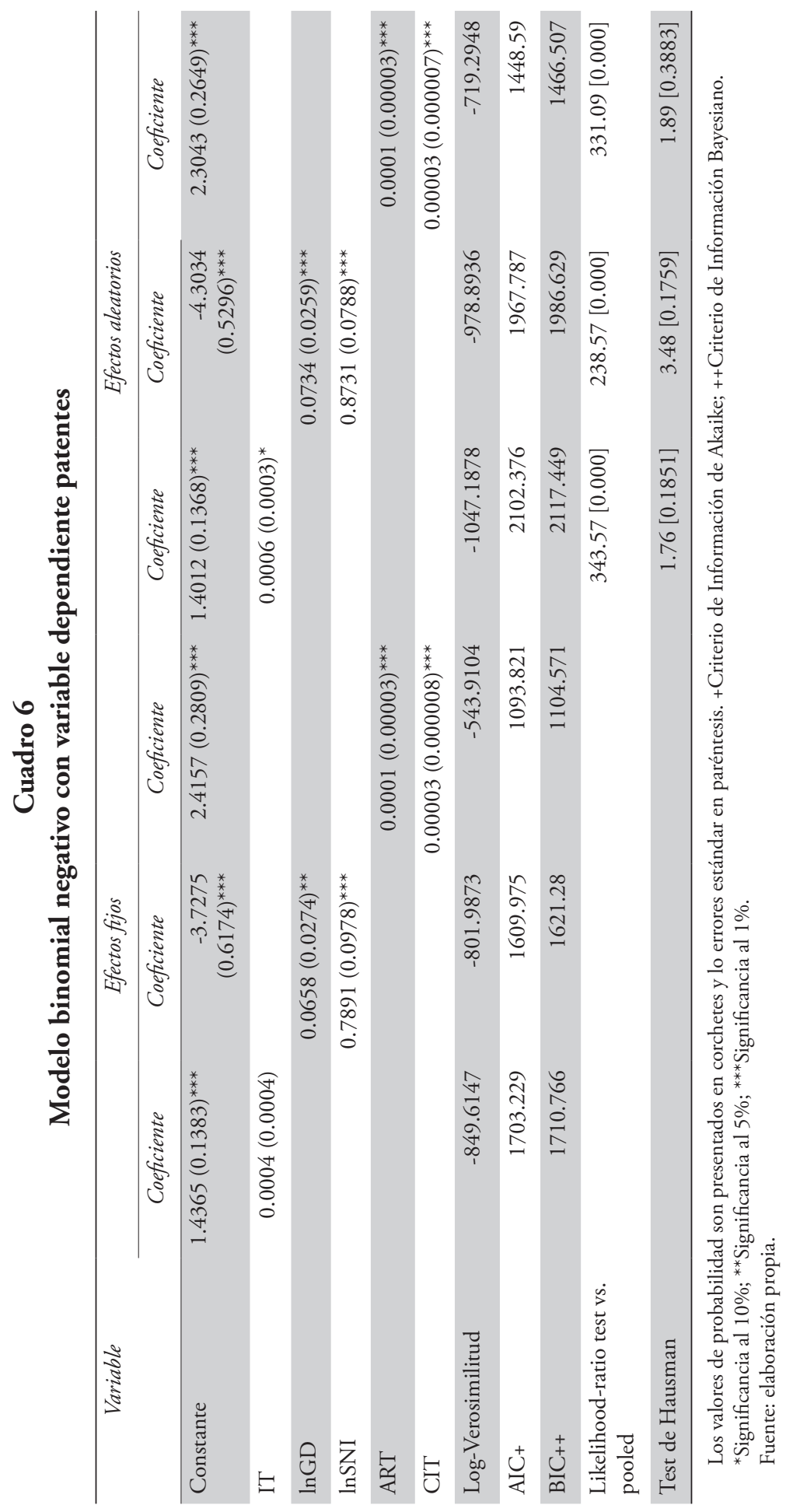




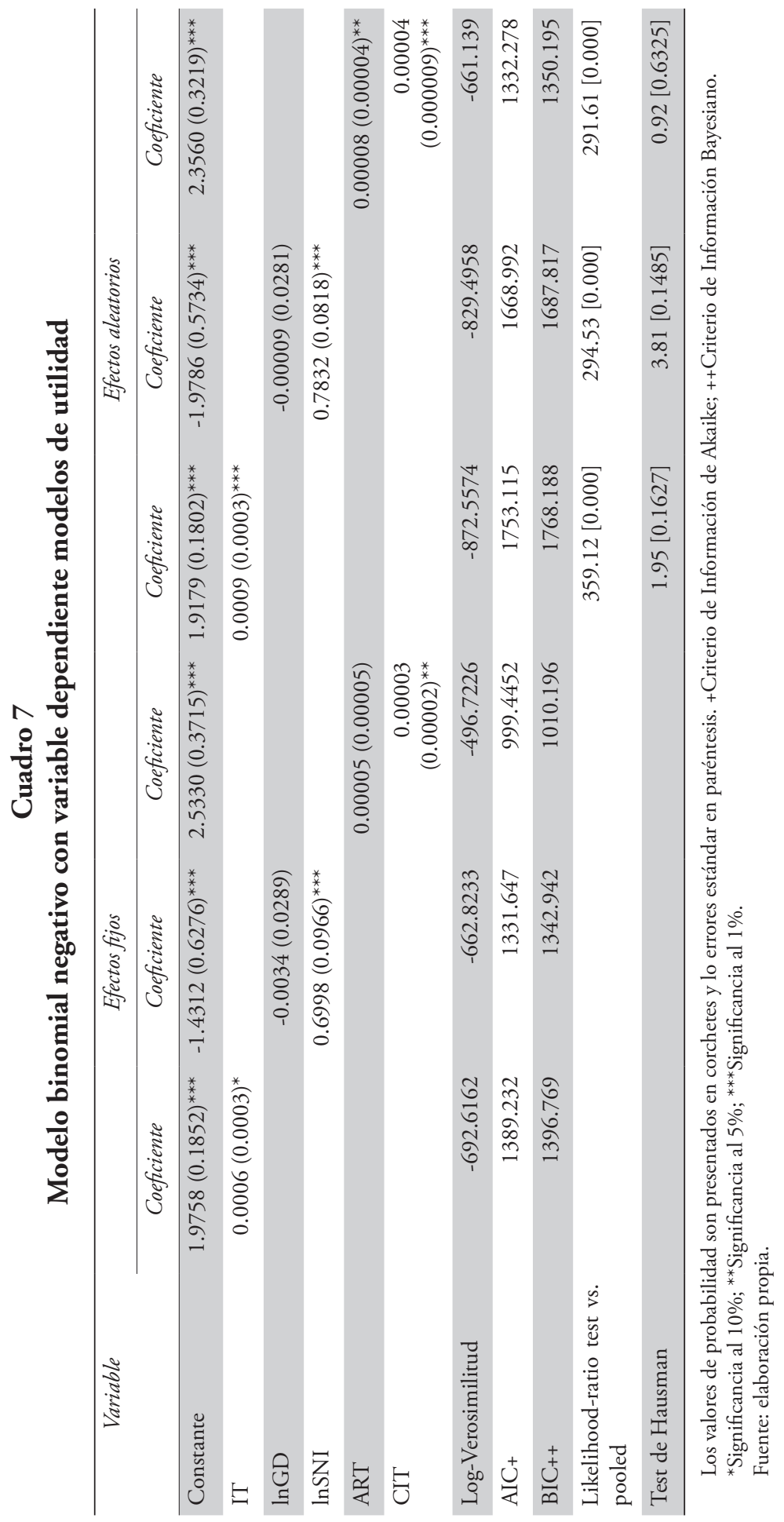




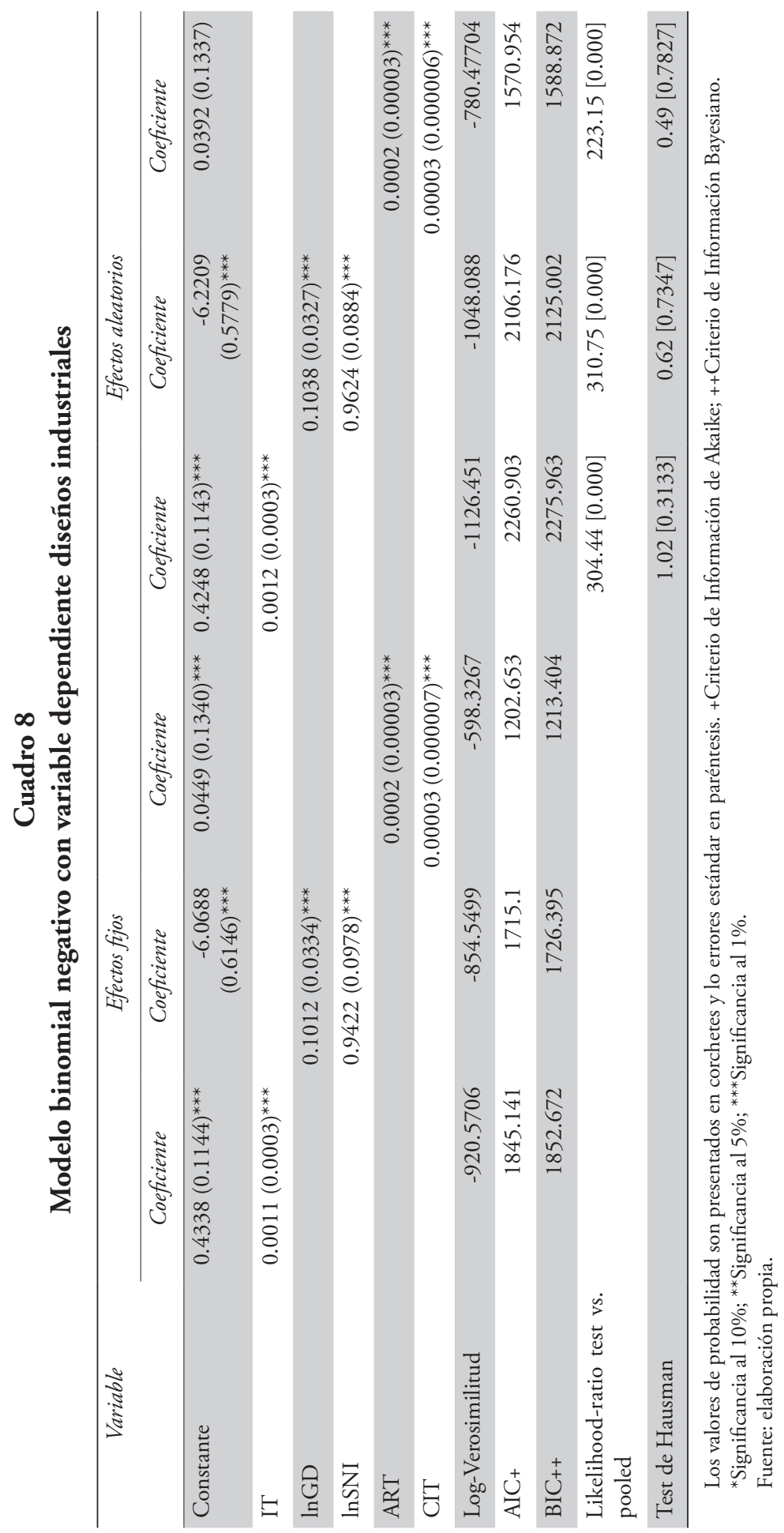


las mismas razones, constituyendo otro modelo. De la misma manera, también los artículos y citas fueron segregados para estructurar otro modelo. Los FIV y los índices del número de condición de ambos resultaron por debajo de 10 y de 20 , respectivamente.

Es importante mencionar que las regresiones de datos de conteo no son lineales. Cuando están presentes variables continuas, los coeficientes se interpretan como la respuesta del cambio proporcional en la media condicional de la variable explicada (recuento esperado) ante un cambio unitario en una variable independiente determinada, refiriéndose a semielasticidades; cuando las variables explicativas son transformadas a logaritmos se describen como elasticidades.

Al comenzar la búsqueda de información tecnológica se muestra evidencia de una relación positiva con los tres tipos de propiedad intelectual, sugiriendo que el aumento de una unidad en la variable explicativa incrementa las patentes en $0.06 \%$, los modelos de utilidad en $0.09 \%$ y los diseños industriales en $0.12 \%$.

Esto es señal de que recurrir a herramientas como la indagación de la búsqueda tecnológica, que son promovidas por el IMPI para proteger los resultados de los trabajos de los inventores, tiene como finalidad mejorar la capacidad de estos importantes actores del ecosistema de innovación; por un lado, les permite comprender el estado actual de la técnica y, por el otro, identificar las áreas de oportunidad que se presentan en el mercado para su potencial comercialización.

En conjunto, estos dos aspectos le proporcionan una mayor fortaleza a la redacción de las solicitudes detallando claramente los rasgos que las diferencian. La aportación concuerda con los estudios de DeGroote y Marx (2013) y Foukolaei et al. (2018), que abordan el efecto de la información tecnológica en la creación de productos, procesos o el desempeño de una organización.

El gasto en investigación y desarrollo sólo resultó estadísticamente significativo en las patentes y los diseños industriales, indicando las elasticidades de que un incremento de $1 \%$ en este desembolso eleva en $7.3 \%$ y $10.4 \%$ estas dos figuras de propiedad intelectual, respectivamente.

Su signo es acorde con la teoría económica, donde se explica que funciona como dotación de insumo de la creación de ideas y con la literatura que puntualiza la parte empírica (Hausman et al., 1984; Cozza y Schettino, 2015). Sin embargo, en los estudios de Hu y Mathews (2008) desarrollado para China, Cowan y Zinovyeva (2013) elaborado para Italia, demostraron que el gasto en investigación y desarrollo de origen privado es el determinante de la producción de patentes; incluso resultando el gasto público con signo negativo en el primer trabajo. 
En el primer caso, la explicación encontrada por los autores a propósito del impacto negativo se debe a una ineficiencia y a una desalienación por parte del gasto, mientras que en el segundo caso, el gasto privado y la investigación académica local se han convertido en sustitutos del proceso de innovación.

Aquellos adscritos al SNI también exhibieron un vínculo directo que es coherente con la literatura, donde personal perteneciente al sector de ciencia y tecnología tiene una fuerte propensión a innovar, plasmándose en elasticidades de $0.87,0.78$ y 0.96 . Esto es un reflejo de que la mayor fracción de gasto en investigación y desarrollo tiene origen público. El auspicio por esta vía proviene de las diversas convocatorias de ciencia y tecnología a las que concursan los diversos agremiados del SNI para entrar, mantenerse o promoverse dentro del sistema, con la finalidad de financiar sus proyectos de investigación.

Su culminación trae como consecuencia resultados relevantes que son susceptibles de protección por cualquier figura de propiedad intelectual y de futura comercialización, realizándose a una escala baja. Los resultados son consistentes con los estudios internacionales (Furman et al., 2002; Malva y Carree, 2013; Cozza y Schettino, 2015), que incorporan en el análisis la variable personal científico y tecnológico y los trabajos nacionales que introducen los miembros del SNI (Calderón-Martínez, 2014).

La difusión de la ciencia y la tecnología, a través de la generación de conocimiento, cristalizada en la producción de artículos científicos es un elemento esencial, ya que es un importante "semillero" de una de las etapas del proceso de transferencia de tecnología, como es la creación de ideas que se concretan en la escritura y el trámite de la solicitud ante instituciones especializadas en propiedad intelectual (Finston, 2007).

Los resultados fueron cotejados con la evidencia internacional, la cual sugiere que las publicaciones afectan positivamente en la propiedad intelectual (Carayol, 2007; Stephan et al., 2007; Cowan y Zinovyeva 2013). En México, Calderón-Martínez (2014) hace una distinción entre artículos indexados a International Scientific Indexing (ISI) y artículos indexados a Scopus y encuentra que el primero tuvo signo positivo y el otro no fue estadísticamente significativo, atribuyéndose a que la calidad de la investigación impacta notablemente en la generación de patentes. Otro trabajo, como el de Hernández y Díaz (2007), encuentran que los artículos y las tesis impactan positivamente en las patentes con rendimientos decrecientes, debido a que no resultan fundamentales en el corto plazo en la producción de innovaciones.

Los artículos científicos más referenciados advierten que tienen un nexo positivo con la actividad innovadora, demostrando que su trascendencia en el ámbito académico es crucial para que al inventor lo orienten 
sobre cuáles son las problemáticas primordiales a resolver y que puede desencadenar posteriores fases de investigación dirijidas a la obtención de un desarrollo tecnológico.

Los hallazgos concuerdan con la investigación de Murray y Stern (2007), basada en una muestra de 340 artículos científicos publicados entre 1997 y 1999 en la revista Nature Biotechnology. En este estudio mencionan los autores que, en la mitad de los artículos, el conocimiento es protegido a través de patentes concedidas en Estados Unidos.

En México, los resultados son acordes con los estudios de CalderónMartínez (2014), Calderón-Martínez y García-Quevedo (2013), quienes señalan que los artículos indexados en revistas con factor de impacto son los más referenciados y sinónimo de calidad en la investigación de la propensión a patentar, mientras que sus estimaciones realizadas considerando citas Scopus mostraron un signo negativo.

\section{Conclusiones}

En general, como primer trabajo realizado en México, se cumple el objetivo de mostrar evidencia empírica sobre la relación positiva que guardan a nivel de entidad federativa los indicadores científicos y las búsquedas tecnológicas con las figuras de propiedad intelectual. En la hipótesis formulada se verificó que los estados mexicanos que dedican mayores esfuerzos a investigación y desarrollo, expresado en gasto, productos científicos y búsquedas tecnológicas son más propensos a producir propiedad intelectual.

Los diseños industriales mostraron las magnitudes más altas en los coeficientes de la regresión, corroborando que son los más solicitados por los inventores. En el futuro, estos resultados pueden ir acompañados con las modificaciones que se realizaron a la Ley de Propiedad Intelectual, que entró en vigor el 24 de abril de 2018 , en la que se menciona que los diseños industriales serán otorgados por cinco años y serán renovables hasta 25 años.

La primera limitación que presenta este trabajo es que los hallazgos de las búsquedas de información tecnológica pueden estar subestimados porque solamente consideran información registrada de los servicios que ofreció el IMPI en el periodo estudiado; no contempla las búsquedas tecnológicas realizadas por las Oficinas de Transferencia de Tecnología (OTT), Centros de Patentamiento u otro agente económico, las cuales no están contabilizadas oficialmente.

Otra limitación que presenta este estudio es que la base de datos no permite discernir entre revistas que pertenecen a International Scientific 
Indexing (ISI) y que se encuentran en el Journal Citations Reports (JCR), indexadas a Conacyt, a Scopus o arbitrados.

En investigaciones futuras sería importante relacionar cada una de las modalidades de propiedad intelectual con cada una de las categorías de indexación, ya que las revistas que cuentan con factor de impacto más alto son las que tienen mayores posibilidades de citación. A nivel global, el criterio del factor de impacto es muy importante porque determina las evaluaciones de las instituciones académicas y de los investigadores; se decide el otorgamiento de fondos a los proyectos, marcando tendencias en las líneas de investigación (Aranda, 2009).

Otra investigación posterior que se podría llevar cabo tendría que ver con la medición de los impactos de los proyectos que han sido beneficiados en la convocatoria del Programa de Estímulos a la Innovación (PEI) de Conacyt, dado que algunos de los resultados de este tipo de proyectos pueden ser potenciales candidatos a protegerse con algunas de las figuras de propiedad intelectual.

Por último, la política de ciencia y tecnología en México debe orientarse a fortalecer los vínculos entre el sector académico, el sector productivo, el gobierno y la sociedad para que se potencialicen los esfuerzos dedicados a la generación de conocimiento y de innovación.

Esta es la ruta para que la nación trascienda hacia una economía basada en el conocimiento, en la cual sus frutos permitirían el mejoramiento del bienestar de la población. La política que ha venido implementando Conacyt, como la creación de las OTT, es un buen comienzo, ya que una de sus funciones principales es la de servir como traductor de las necesidades del sector productivo y la oferta tecnológica del gremio académico. Sin embargo, en el largo plazo, llegará el momento de evaluar esta política. En la actualidad, otra política que se está ejecutando dentro de la evaluación de los afiliados del SNI es la consideración de los desarrollos tecnológicos. Esta medida es de enorme relevancia porque puede ser un detonador del proceso de innovación.

\section{Fuentes consultadas}

Aghion, Philippe y Peter Howitt (1992), "A model of growth through creative destruction”, Econometrica, 60 (2), Wiley, Nueva Jersey, Estados Unidos de América, pp. 323-351.

Amigo, Jorge (2009), "Scientific and technological policy in Mexico and intellectual property”, en José Mario Martínez-Piva (ed.), Knowledge generation and protection. Intellectual property, innova- 
tion and economic development, Naciones Unidas, Nueva York, Estados Unidos de América, pp. 291-304.

Aranda, Armando (2009), "La ciencia posmoderna y el factor de impacto", Ciencia Ergo Sum, 16 (2), Universidad Autónoma del Estado de México, Toluca, Estado de México, pp. 17-22.

Arun, Korhan y Durmus Yildirim (2017), "Effects of foreign direct investment on intellectual property, patents and R\&D", Munich Personal RePEC Archive (MPRA), paper núm. 80470, University Library of Munich, Alemania, pp. 1-16.

Azagra-Caro, Joaquín (2014), "Determinants of national patent ownership by public research organisations and universities", The Journal of Technology Transfer, 39 (6), Springer, Nueva York, Estados Unidos de América, pp. 898-914.

Baglieri, Daniela y Fabrizio Cesaroni (2013), "Capturing the real value of patent analysis for R\&D strategies", Technology Analysis and Strategic Management, 25 (8), Routlegde Journals, Abingdon, Inglaterra, pp. 971-986.

Blazseck, Szabolcs y Álvaro Escribano (2010), "Knowledge spillovers in US patents: A dynamic patent intensity model with secret common innovation factors", Journal of Econometrics, 159 (1), Elsevier Science, Lausanne, Suiza, pp. 14-32.

Boix, Rafael y Vittorio Galletto (2009), "Innovation and industrial districts: a first approach to the measurement and determinants of the I-District Effect", Regional Studies, 43 (9), Routledge Journals, Abingdon, Inglaterra, pp. 1117-1133.

Boldrin, Michele y David Levine (2013), "What's intellectual property good for?”, Revue économique, 64 (1), Journal STORage, París, Francia, pp. 29-53.

Brack, Hans-Peter (2009), Utility models and their comparison with patents and implications for the US intellectual property law system, Boston College Intellectual Property and Technology Forum, Boston, Estados Unidos de América, pp. 1-15. 
Breschi, Stefano y Camilla Lenzi (2016), "Co-invention networks and inventive productivity in US cities", Journal of Urban Economics, núm. 92, Elsevier Science, San Diego, Estados Unidos de América, pp. 66-75.

Brolund, Johan y Robert Lundmark (2014), "Bioenergy innovations and their determinants: a negative binominal count data analysis", Drewno, 57 (192), Institute Technology Drewna, Posnan, Polonia, pp. 41-61.

Calderón-Martínez, Guadalupe (2014), "Patentes en instituciones de educación superior en México", Revista de la Educación Superior, XLIII (2), Asociación Nacional de Universidades e Instituciones de Educación Superior, Ciudad de México, México, pp. 37-56.

Calderón-Martínez, María y José García-Quevedo (2013), "Knowledge transfer and university patents in Mexico", Revista Latinoamericana de Administración, 26 (1), Emerald, Bingley, Inglaterra, pp. 33-60.

Cameron, Colin y Pravin Trivedi (2005), Microeconometrics. Methods and applications, Cambridge University Press, Cambridge, Reino Unido, pp. 1034.

Carayol, Nicolas (2007), "Academic incentives, research organization, and patenting at a large French university", Economics of Innovation and New Technology, 16 (2), Routlegde Journals, Abingdon, Inglaterra, pp. 119-138.

Carree, Martin, Roberta Piergiovanni, Enrico Santarelli e Ingrid Verheul (2015), "Factors favoring innovation from a regional perspective: a comparison of patents and trademarks", International Entrepreneurship and Management Journal, 11 (4), Springer, Nueva York, Estados Unidos de América, pp. 793-810.

Carvalho, Marly, André Fleury y Ana Lopes (2013), “An overview of the literature on technology roadmapping (TRM): Contributions and trends", Technological Forecasting and Social Change, 80 (7), Elsevier Science, Nueva York, Estados Unidos de América, pp. 1418-1437.

Chang, Yuan-Chieh, Phil Yang y Ming-Huei Chen (2009), "The determinants of academic research commercial performance: towards 
an organizational ambidexterity perspective", Research Policy, 38 (6), Elsevier, Amsterdam, Holanda, pp. 936-946.

Cowan, Robin y Natalia Zinovyeva (2013), "University effects on regional innovation", Research Policy, 42 (3), Elsevier, Amsterdam, Holanda, pp. 788-800.

Cozza, Claudio y Francesco Schettino (2015), "Explaining the patenting propensity: a regional analysis using EPO-OECD Data", en Chiara Mussida y Francesco Pastore (eds.), Geographical Labor Market Imbalances: Recent Explanations and Cures, Springer, Nueva York, Estados Unidos de América, pp. 219-236.

DeGroote, Sharon y Thomas Marx (2013), "The impact of IT on supply chain agility and firm performance: an empirical investigation", International Journal of Information Management, 33 (6), Elsevier Science, Ámsterdam, Holanda, pp. 909-916.

Dettmann, Eva, Iciar Dominguez Lacasa, Jutta Günther y Björn Jindra, (2013), "Determinants of foreign technological activity in German regions - A count model analysis of transnational patents (1996-2009)", ECONSTOR, working paper núm. 12/2013IWH discussion papers, Leibniz Information Centre for Economics, Kiel, Alemania, pp. 1-32.

Díaz, Maidelyn (2008), "La propiedad industrial y los sistemas de patentes en el mundo de la información", Revista Cubana de Información en Ciencias de la Salud, 18 (6), El Centro Nacional de Información de Ciencias Médicas, La Habana, Cuba, pp. 1-11.

Feeny, Simon y Mark Rogers (2003), "Innovation and performance: Benchmarking Australian Firms", The Australian Economic Review, 36 (3), Wiley, Melbourne, Australia, pp. 253-264.

Finston, Susan (2007), "Technology transfer snapshots from middle-income countries: creating socio-economic benefits through innovation", en Anatole Krattiger, Richard Mahoney, Lita Nelsen, Jennifer Thomson, Alan Benett, Kanikaram Satyanarayana, Gregory Graff, Carlos Fernandez, Stanley Kowalski (eds.), Intellectual property management in health and agricultural innovation: a handbook of best practices, volume one, Centre for the Management of Intellectual Property in Health Research and Development-Public Intellectual 
Property Resource for Agriculture, Oxford-Los Ángeles California, Inglaterra-Estados Unidos de América, pp. 197-205.

Foukolaei, Parvaneh, Mehrdad Madhoushi, Hasanali Aghajani y Mahmod Yahyazadehfar (2018), "Developing a local model to evaluate the impact of information technology capabilities on the performance of pharmaceutical firms using the mediating role of supply chain approach (Case study: Pharmaceutical Firms in Iran)", Journal of Information Technology Management, 9 (4), Universidad de Baltimore, Baltimore, Estados Unidos de América, pp. 829-850.

Fundación Cotec (2003), Nuevos mecanismos de transferencia de tecnología. Debilidades y oportunidades del sistema español de transferencia de tecnologia, Fundación COTEC para la Innovación Tecnológica, Madrid, España, pp. 190.

Furman, Jeffrey, Michael Porter y Scott Stern (2002), "The determinants of national innovative capacity”, Research Policy, 31 (6), Elsevier, Ámsterdam, Holanda, pp. 899-933.

García, Rodolfo (2017), "Patentamiento universitario e innovación en México, país en desarrollo: teoría y política", Revista de la Educación Superior, 46 (184), Asociación Nacional de Universidades e Instituciones de Educación Superior, Ciudad de México, México, pp. 77-96.

Germán-Soto, Vicente y Luis Gutiérrez (2015), “A standardized coefficients model to analyze the regional patents activity: evidence from the Mexican states", Journal of the Knowledge Economy, 6 (1), Springer, Nueva York, Estados Unidos de América, pp. 72-89.

Germán-Soto, Vicente, Luis Gutiérrez y Sandra Tovar (2009), "Factores y relevancia geográfica del proceso de innovación regional en México, 1994-2006”, Estudios Económicos, 24 (2), El Colegio de México, Ciudad de México, México, pp. 225-248.

Glauber, Johanna, Jutta Wollersheim, Philipp Sandner e Isabell Welpe (2015), "The patenting activity of German universities", Journal of Business Economics, 85 (7), Springer, Nueva York, Estados Unidos de América, pp. 719-757. 
González-Pernía, José, Graciela Kuechle e Iñaki Peña-Legazkue (2013), "An assessment of the determinants of University Technology Transfer", Economic Development Quarterly, 27 (1), Sage, Thousand Oaks, Estados Unidos de América, pp. 6-17.

Gracia, Maximiliano y Eduardo Moctezuma (2016), “¿Hay impacto del capital humano en la innovación tecnológica de México?”, Revista OIKOS, 20 (41), Universidad Católica Silva Henríquez, Santiago, Chile. pp. 49-68.

Greene, William (2003), Econometric Analysis, Prentice Hall, Nueva Jersey, Estados Unidos de América.

Grossman, Gene y Elhanan Helpman (1991), Innovation and growth in the global economy, MIT Press Cambridge, Massachusetts, Estados Unidos de América.

Griliches, Zvi (1979), "Issues in assessing the contribution of research and development to productivity growth", Bell Journal of Economics, 10 (1), Wiley-Blackwell, Nueva Jersey, Estados Unidos de América, pp. 92-116.

Griliches, Zvi (1990), "Patent statistics as economic indicators: a survey", Journal of Economic Literature, 28 (4), American Economic Association, Nashville, Estados Unidos de América, pp. 1661-1707.

Gujarati, Damodar (2004), Basic econometrics, McGraw-Hill, Nueva York, Estados Unidos de América.

Guzmán, Alenka, Edgar Acatitla y Thalia Vázquez (2016), "Propensity toward industry-science links across Mexico's technological sectors: an analysis of patents, 1980-2013", EconoQuantum, 13 (1), Universidad de Guadalajara, Guadalajara, México, pp. 125-157.

Hausman, Jerry, Bronwyn Hall y Zvi Griliches (1984), "Econometric models for count data with an application to the patents-r \& d relationship", Econometrica, 52 (4), Wiley, Nueva Jersey, Estados Unidos de América, pp. 909-938.

Hernández, Sergio y Eliseo Díaz (2007), "La producción y el uso del conocimiento en México y su impacto en la innovación: análisis regional de las patentes solicitadas", Análisis Económico, XII (50), 
Universidad Autónoma Metropolitana, Ciudad de México, México, pp. 185-217.

Hu, Albert (2010), "Propensity to patent, competition and China's foreign patenting surge", Research Policy, 39 (7), Elsevier, Ámsterdam, Holanda, pp. 985-993.

Hu, Mei-Chih y John Mathews (2008), "China's national innovative capacity”, Research Policy, 37 (9), Elsevier, Ámsterdam, Holanda, pp. 1465-1479.

Huang, Can y Jojo Jacob (2014), "Determinants of quadic patenting: market access, imitative threat, competition and strength of intellectual property rights", Technological Forecasting and Social Change, núm.85, Elsevier Science, Nueva York, Estados Unidos de América, pp. 4-16.

Huang, Kuo-Feng y Tsung-Chi Cheng (2015), "Determinants of firms' patenting or not patenting behaviors", Journal of Engineering and Technology Management, núm. 36, Elsevier Science, Nueva York, Estados Unidos de América, pp. 52-77.

Hudson, John y Alexandru Minea (2013), "Innovation, intellectual property rights, and economic development: a unified empirical Investigation”, World Development, núm. 46, Pergamon-Elsevier Science, Oxford, Inglaterra, pp. 66-78.

IMPI (Instituto Mexicano de la Propiedad Industrial), (2018), Guia del usuario de diseños industriales, IMPI, Ciudad de México, México.

IMPI (Instituto Mexicano de la Propiedad Industrial), (2017), IMPI en Cifras 2017, IMPI, Ciudad de México, México.

Jeong, Yujin y Byungun Yoon (2015), “Development of patent roadmap based on technology roadmap by analyzing patterns of patent development", Technovation, núm. 39-40, Elsevier, Ámsterdam, Holanda, pp. 37-52.

Kim, Yee, Keun Lee, Walter Park y Kineung Choo (2012), "Appropriate intellectual property protection and economic growth in countries at different levels of development", Research Policy, 41 (2), Elsevier, Ámsterdam, Holanda, pp. 358-375. 
Kivimäki, Mika, Hannakaisa Länsisalmi, Marko Elovainio, Armo Heikkilä, Kari Lindström, Risto Harisalo, Kari Sipila y Leena Puolimatka (2000), "Communication as a determinant of organizational innovation", R and D Management, 30 (1), Wiley, Nueva Jersey, Estados Unidos de América, pp. 33-42.

Kurman, Melba (2011), "An index-based measure of University Technology Transfer", International Journal of Innovation Science, 3 (4), Emerald, Bingley, Inglaterra, pp. 167-176.

Lee, Sungjoo, Seonghoon Lee, Hyeonju Seol y Yongtae Park (2008), "Using patent information for designing new product and technology: keyword based technology roadmapping", $R$ and $D$ Management, 38 (2), Wiley, Nueva Jersey, Estados Unidos de América, pp. 169-188.

Livotov, Pavel (2015), “Using patent information for identification of new product features with high market potential", Procedia Engineering, núm.131, Elsevier, Ámsterdam, Holanda, pp. 1157-1164.

Malva, Antonio y Martin Carree (2013), “The spatial distribution of innovation: evidence on the role of academic quality for seven European countries", Economics of Innovation and New Technology, 22 (6), Routlegde Journals, Abingdon, Inglaterra, pp. 601-618.

Martínez-Piva, José (2009), "Protection of intellectual property rights, innovation and development", en José Mario Martínez-Piva (ed.), Knowledge deneration and protection: intellectual property, innovation and economic development, Naciones Unidas, Nueva York, Estados Unidos de América, pp. 27-55.

Mendonca de, Vera (2012), "Investigación y desarrollo: la formación de los recursos humanos en Brasil”, Universidades, núm. 52, Unión de Universidades de América Latina y el Caribe, Ciudad de México, México, pp. 18-26.

Murray, Fiona y Scott Stern (2007), "Do formal intellectual property rights hinder the free flow of scientific knowledge? An empirical test of the anti-commons hypothesis", Journal of Economic Behavior and Organization, 63 (4), Elsevier, Ámsterdam, Holanda, pp. 648-687. 
Nambisan, Satish (2003), "Information systems as a reference discipline for new product development”, MIS Quarterly, 27 (1), Universidad de Minnesota, Minneapolis, Estados Unidos de América, pp. 1-18.

Nikzad, Rashid (2012), "Transfer of technology to Canadian manufacturing industries through patents", Australian Economic Papers, 51 (4), Wiley, Nueva Jersey, Estados Unidos de América, pp. 210-227.

OCDE (Organización para la Cooperación y el Desarrollo Económicos) (2004), Patents and innovation: trends and policy challenges, OCDE, París, Francia.

OCDE (Organización para la Cooperación y el Desarrollo Económicos) (2015), Estudios Económicos de la OCDE México: Visión General, OCDE, Ciudad de México, México.

OCDE-Eurostat (Organización para la Cooperación Desarrollo Económicos-European Statistical System) (2006), Guí para la recogida e interpretación de datos sobre innovación, OCDE-EUROSTAT, París, Francia.

OMPI (Organización Mundial de la Propiedad Intelectual) (2013), Las patentes: fuente de información tecnológica, OMPI, Ginebra, Suiza.

OMPI (Organización Mundial de la Propiedad Intelectual) (2015), “Curso general sobre propiedad intelectual (DL-101)”, OMPI, Ciudad de México, México, <http://www.wipo.int/academy/es/courses/ distance_learning/dl101.html>, 6 de diciembre de 2016.

Ortega-Argilés, Raquel y Rosina Moreno (2009), "Evidence on the role of ownership structure on firms' innovative performance", Investigaciones Regionales, núm. 15, Asociación Española de Ciencia Regional, Barcelona, España, pp. 231-250.

Ozbugday, Fatih y Erik Brouwer (2012), “Competition law, networks and innovation", Applied Economics Letters, núm. 19, Routlegde Journals, Abingdon, Inglaterra, pp. 775-778. 
Papageorgiadis, Nikolaos y Abhijit Sharmab (2016), "Intellectual property rights and innovation: a panel analysis", Economics Letters, núm. 141, Routlegde Journals, Abingdon, Inglaterra, pp. 70-72.

Pavitt, K. (1985), "Patent statistics as indicators of innovative activities: possibilities and problems", Scientometrics, 7 (1-2), Springer, Dordrecht, Holanda, pp. 77-99.

Rizzo, Ugo y Laura Ramaciotti (2014), "The determinants of academic patenting by Italian universities", Technology Analysis and Strategic Management, 26 (4), Routlegde Journals, Abingdon, Inglaterra, pp. 469-483.

Rogers, Everett, Jing Yin y Joern Hoffmann (2000), "Assessing the effectiveness of technology transfer offices at U.S. research universities", The Journal of the Association of University Technology Managers, núm. 12, Association of University Technology Managers, Washington, Estados Unidos de América, pp. 47-80.

Romer, Paul (1990), "Endogenous technological change", Journal of Political Economy, 98 (5), University Chicago Press, Chicago, Estados Unidos de América, pp. S71-S102.

Stephan, Paul, Shiferaw Gurmu, Albert Sumell y Grant Black (2007), "Who's patenting in the university? Evidence from the survey of doctorate recipients", Economics of Innovation an New Technology, 16 (2), Routlegde Journals, Abingdon, Inglaterra, pp. 71-99.

Sun, Yifei (2003), "Determinants of foreign patents in China”, World Patent Information, 25 (1), Elsevier Science, Amsterdam, Holanda, pp. 27-37.

Sun, Yifei y Debin Du (2010), "Determinants of industrial innovation in China: evidence from its recent economic census", Technovation, 30 (9-10), Elsevier, Ámsterdam, Holanda, pp. 540-550.

Suthersanen, Uma (2006), Utility models and innovation in developing countries, International Centre for Trade and Sustainable Development-United Nations Conference on Trade and Development, Geneva, Suiza. 
Torres-Preciado, Víctor, Mayrén Polanco Gaytán y Miguel Tinoco Zermeño (2014), "Technological innovation and regional economic growth in Mexico: a spatial perspective", Annals of Regional Science, 52 (1), Springer, Nueva York, Estados Unidos de América, pp. 183-200.

Thakur, Sudhir y Edward Malecki (2015), "Regional determinants of research and development institutions in India", GeoJournal, 80 (4), Springer, Dordrecht, Holanda, pp. 533-554.

Ulku, Hulya (2004), "R\&D, innovation, and economic growth: an empirical analysis", working paper, núm. 04/185, International Monetary Fund, Washington, Estados Unidos de América.

Valdivia, Marcos (2007), "Heterogeneidad espacial, convergencia y crecimiento regional en México", ponencia para el XVII Coloquio de Economía Matemática y Econometría, 21-25 de mayo, Universidad de Quintana Roo, Quintana Roo, México.

Varga, Attila, Dimitrios Pontikakis y George Chorafakis (2014), "Metropolitan Edison and cosmopolitan Pasteur? Agglomeration and interregional research network effects on European R\&D productivity", Journal of Economic Geography, 14 (2), Oxford University Press, Oxford, Inglaterra, pp. 229-263.

Winkelmann, Rainer y Klaus Zimmermann (1991), "A new approach for modelling economic count data", Economics Letters, 37 (2), Routlegde Journals, Abingdon, Inglaterra, pp. 139-143.

WIPO (World Intellectual Property Organization) (1999), WIPO regional seminar on the protection of intellectual property and the commercialization of inventions, WIPO-Ministry of Supply and Home Trade-Association of Syrian Inventors, Alepo, Siria.

Yang, Chih-Hai y Nai-Fong Kuo (2008) "Trade-related influences, foreign intellectual property rights and outbound international patenting", Research Policy, 37 (3), Elsevier, Ámsterdam, Holanda, pp. 446-459.

Zeebroeck, Nicolas van, Bruno van Pottelsberghe de la Potterie y Dominique Guellec (2009), "Claiming more: the increased volumino- 
sity of patent applications and its determinants", Research Policy, 38 (6), Elsevier, Ámsterdam, Holanda, pp. 1006-1020.

Recibido: 28 de febrero 2018. Revisado: 3 de mayo 2018. Aceptado: 31 de mayo 2018.

Marco Antonio Almendarez Hernández. Doctor en Ciencias Marinas y Costeras con orientación en manejo sustentable por la Universidad Autónoma de Baja California Sur (UABCS); maestro en Economía aplicada por El Colegio de la Frontera Norte (El COLEF). Actualmente es economista de la Oficina de Propiedad Intelectual y Comercialización de Tecnología (OTT/ CEPAT) del Centro de Investigaciones Biológicas del Noroeste (CIBNOR); adicionalmente es profesor-investigador del Departamento de Economía de la Universidad Autónoma de Baja California Sur (UABCS). Es miembro del Sistema Nacional de Investigadores, nivel I. Sus líneas de investigación son economía aplicada, economía ambiental, economía de los recursos naturales, economía de la innovación, economía del conocimiento y economía sectorial. Entre sus últimas publicaciones destacan: "Residential water demand in a Mexican Biosphere Reserve: evidence of the effects of perceived price”, Water, 8 (10), Multidisciplinary Digital Publishing Institute, Basilea, Suiza, pp. 1-14 (2016); "Propuesta de cuotas para conservación de un área natural protegida de México", Perfiles Latinoamericanos, 24 (47), Facultad Latinoamericana de Ciencias Sociales Sede México (FLACSO-México), Ciudad de México, México, pp. 95-120 (2016); "Demanda de agua de uso comercial en la Reserva de La Biosfera El Vizcaíno: una estimación con datos de panel, Nova Scientia, 7 (15), Universidad De La Salle Bajío, León, México, pp. 553-576, (2015); "Determinantes en el consumo de atún en México aplicando modelos de elección ordenada", Interciencia, 40 (6), Interciencia, Caracas, Venezuela, pp. 390-396 (2015). 